\title{
Electrochemical Behaviors and Electrochemical Determination of 1-naphthaleneacetic Acid at an Ionic Liquid Modified Carbon Paste Electrode
}

\author{
Cheng-Qian Duan, ${ }^{\text {a,b }}$ Yan-Mei Zhang, ${ }^{\text {a }}$ and Zuo-Ning Gao ${ }^{\mathrm{a}, *}$ \\ ${ }^{a}$ Key Lab of Energy Sources and Chemical Engineering, College of Chemistry and Chemical Engineering, \\ Ningxia University, Yinchuan, 750021, People's Republic of China \\ ${ }^{\mathrm{b}}$ Research Section of Higher Vocational College, Ningxia Medical University, Yinchuan, 750004, \\ People's Republic of China
}

RECEIVED APRIL 19, 2011; REVISED JUNE 13, 2011; ACCEPTED JUNE 13, 2011

\begin{abstract}
Electrochemical behaviors of 1-naphthaleneacetic acid (NAA) at an ionic liquid $N$-butylpyridinium hexafluorophosphate $\left(\mathrm{BPPF}_{6} / \mathrm{CPE}\right)$ modified carbon paste electrode was investigated by cyclic voltammetry (CV) and further used for NAA sample determination. The experimental results showed that NAA at both the bared and the modified electrode showed an irreversible oxidation process, but at the modified electrode it exhibited an enhanced electrochemical response towards its electrochemical oxidation. $\mathrm{BPPF}_{6} / \mathrm{CPE}$ was characterized by electrochemical impedance spectroscopy (EIS). At the same time, the electrochemical kinetic parameters were determined at both electrodes. The oxidation peak currents increased linearly with NAA concentration range from $2.0 \times 10^{-5}$ to $4.0 \times 10^{-4} \mathrm{~mol} \mathrm{dm}^{-3}$ with the detection limit $(\mathrm{S} / \mathrm{N}=3)$ of $1.690 \times 10^{-7} \mathrm{~mol} \mathrm{dm}^{-3}$ under the optimized experiment conditions. The proposed method was successfully applied for NAA content determination in both commercial formulations and the soil samples. (doi: 10.5562/cca1901)
\end{abstract}

Keywords: 1-naphthaleneacetic acid, ionic liquid modified carbon paste electrode, electrochemistry, electrochemical determination

\section{INTRODUCTION}

1-naphthaleneacetic acid (NAA, structural formula is shown in Figure 1) is naphthalene derivatives used as plant growth regulator to prevent the premature fall of fruit and is also widely used in agriculture, forestry, medical and the other areas. It could stimulate one's skin and mucosa. Once coming into esophagus, it lets people poisoned, and then could cause damage to the liver and the kidney. Therefore, to control its quality, the development of various techniques for its determination is very important. To date, various techniques have been used to determine NAA, including gas chromatography (GC), ${ }^{1}$ high-performance liquid chromatography (HPLC), ${ }^{2}$ spectrofluorimetry, ${ }^{3,4}$ phosphorimetry, ${ }^{5}$ room temperature phosphorescence (RTP) ${ }^{6}$ capillary micelle electrokinetic chromatography (CMEK), ${ }^{7,8}$ and the electrochemical methods. ${ }^{9,10}$ Among the electrochemical ones mentioned above, they mainly discuss the electrochemical behaviors and the determinations of NAA at carbon nanotubes film modified electrode and at ace- tylene black modified glassy carbon electrode. While, its electrochemical behaviors, the electrochemical kinetics and the electrochemical determination at an ionic liquid $N$-butylpyridinium hexafluorophosphate modified carbon paste electrode $\left(\mathrm{BPPF}_{6} / \mathrm{CPE}\right)$ has not been reported yet.

Recently the application of room temperature ionic liquids (RTILs) in the fields of electrochemistry and electroanalytical chemistry had attracted much attention. Room-temperature ILs is an entirely composed of organic cations and various inorganic anions that exist in the liquid state around the room temperature. At present,<smiles>O=C(O)Cc1cccc2ccccc12</smiles>

Figure 1. Structural formula of 1-naphthaleneacetic acid.

\footnotetext{
* Author to whom correspondence should be addressed. (E-mail: gaozn@nxu.edu.cn)
} 


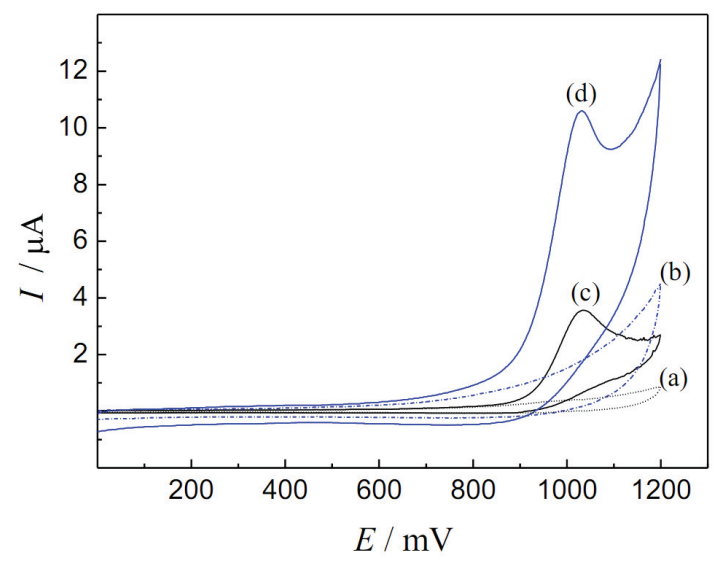

Figure 2. $\mathrm{CV}$ curves of $\mathrm{CPE}$ (a) and $\mathrm{BPPF}_{6} / \mathrm{CPE}$ (b) in 0.10 mol $\mathrm{dm}^{-3} \mathrm{NaClO}_{4}$ in the absence and the presence of $3.0 \times 10^{-4} \mathrm{~mol} \mathrm{dm}^{-3} \mathrm{NAA}$ at $\mathrm{CPE}$ (c) and $\mathrm{BPPF}_{6} / \mathrm{CPE}$ (d). Scan rate: $50 \mathrm{mV} \mathrm{s}^{-1}$.

ILs have attracted more considerable attention for their unique physical and chemical properties, such as wide electrochemical windows, high thermal stability, high conductivity, negligible vapor pressure and adjustability of characteristics via the choice of the anion and cation combination etc. ${ }^{11,12}$ Therefore, RTILs could be used as a modifier or binder to fabricate carbon paste electrodes.

As continuations of our previous work, ${ }^{13-15}$ we chose hydrophobic ionic liquid $N$-butylpyridinium hexafluorophosphate $\left(\mathrm{BPPF}_{6}\right)$ as a modifier to fabricate $\mathrm{BPPF}_{6} / \mathrm{CPE}$ and investigated the electrochemical behaviors and the electrochemical determination of NAA. Its electrochemical kinetic parameters at $\mathrm{BPPF}_{6} / \mathrm{CPE}$, such as charge-transfer coefficient $\alpha$, diffusion coefficient $D$ and the electrode reaction rate constant $k_{\mathrm{f}}$ were determined. Meanwhile, an electrochemical quantitative determination was established which was successfully used for NAA content determination in both the commercial formulations and the real soil samples.

\section{EXPERIMENTAL}

\section{Instrumentation and Reagents}

All electrochemical experiments were carried out by Electrochemistry Workstation CHI660A (CH Instrument, USA). The working electrodes were carbon paste electrode (CPE) and $N$-butylpyridinium hexafluorophosphate $\left(\mathrm{BPPF}_{6}\right)$ modified carbon paste electrode $\left(\mathrm{BPPF}_{6} / \mathrm{CPE}\right)$. A CHI1 15 platinum wire and a CHI150 saturated calomel electrode (SCE) served as the auxiliary electrode and the reference electrode, respectively. All potentials measured and reported in this work were versus a SCE.

1-naphthaleneacetic acid (Batch No. 20081117) was from Shanghai Runjie Chemical Reagent Co., Ltd
(Shanghai, China) and used as received and without further purification. 1-naphthaleneacetic acid samples (Batch No. HNP 51053-E0233) from Sichuan Guoguang Agrochemical Co., Ltd (Sichuan, China), $\mathrm{N}$-butylpyridinium hexafluorophosphate $\left(\mathrm{BPPF}_{6}\right)$ from Chengjie Chemical Reagent Co., Ltd (Shanghai, China, Purity $>99 \%$ ). All solutions were prepared by using deoxygenate and doubly distilled water. All of the other chemicals were of analytical grade and also used as received. All experiments were carried out at room temperature.

\section{Preparation of $\mathrm{CPE}$ and $\mathrm{BPPF}_{6} / \mathrm{CPE}$}

Carbon paste electrode was prepared as the followings: $1.800 \mathrm{~g}$ of graphite and $0.60 \mathrm{~cm}^{-3}$ of paraffin oil were mixed in a mortar to form a homogeneous mixture, and then pressed by hand into the end cavity of a polytetrafluoroethylene (PTFE) cylindrical electrode body and the electrode surface was polished on a piece of weighing paper.

$\mathrm{BPPF}_{6} / \mathrm{CPE}$ was prepared as the followings: $0.6000 \mathrm{~g}$ of $\mathrm{BPPF}_{6}$ was first dissolved in $0.60 \mathrm{~cm}^{-3}$ of $\mathrm{DMF}$, and then added in $1.800 \mathrm{~g}$ of graphite powder in a mortar, ground until DMF totally volatilized, and finally mixed with $0.60 \mathrm{~cm}^{-3}$ of paraffin oil in a mortar to form a homogeneous mixture, and then pressed by hand into the end cavity of a polytetrafluoroethylene (PTFE) cylindrical electrode body and the electrode surface was polished on a piece of weighing paper.

\section{RESULTS AND DISCUSSION}

\section{Cyclic Voltammetric Behaviors of NAA}

The electrochemical behaviors of $3.0 \times 10^{-4} \mathrm{~mol} \mathrm{dm}^{-3}$ NAA in $0.10 \mathrm{~mol} \mathrm{dm}^{-3} \mathrm{NaClO}_{4}$ were investigated at both bared $\mathrm{CPE}$ and $\mathrm{BPPF}_{6} / \mathrm{CPE}$ with scanning rate of $50 \mathrm{mV} \mathrm{s}^{-1}$ in the potential range from 0.40 to $1.20 \mathrm{~V}$. Cyclic voltammogram curves of NAA at both CPE and $\mathrm{BPPF}_{6} / \mathrm{CPE}$ were shown in Figure 2. From the curves in Figure 2, it can be seen that an irreversible oxidation peak on CPE appeared at $1.03 \mathrm{~V}$ with the peak current as $3.254 \times 10^{-6} \mathrm{~A}$ (curve c). While at $\mathrm{BPPF}_{6} / \mathrm{CPE}$, there was a sensitive oxidation peak appeared at $1.03 \mathrm{~V}$ with the peak current as $8.537 \times 10^{-6} \mathrm{~A}$ (curve d). It can be seen that NAA oxidation potentials keep almost constant, and the oxidation peak current at $\mathrm{BPPF}_{6} / \mathrm{CPE}$ was much larger than that at $\mathrm{CPE}$, it was about 3 times larger than that at $\mathrm{CPE}$. Therefore, $\mathrm{BPPF}_{6} / \mathrm{CPE}$ exhibits a much better electrochemical activity towards NAA electrochemical oxidation. This experimental result can be further verified the superiority of $\mathrm{BPPF}_{6} / \mathrm{CPE}$ to $\mathrm{CPE}$, indicating that the use of ILs as a modifier can facilitate the electron transference between NAA and the electrode surface. ${ }^{16}$ 


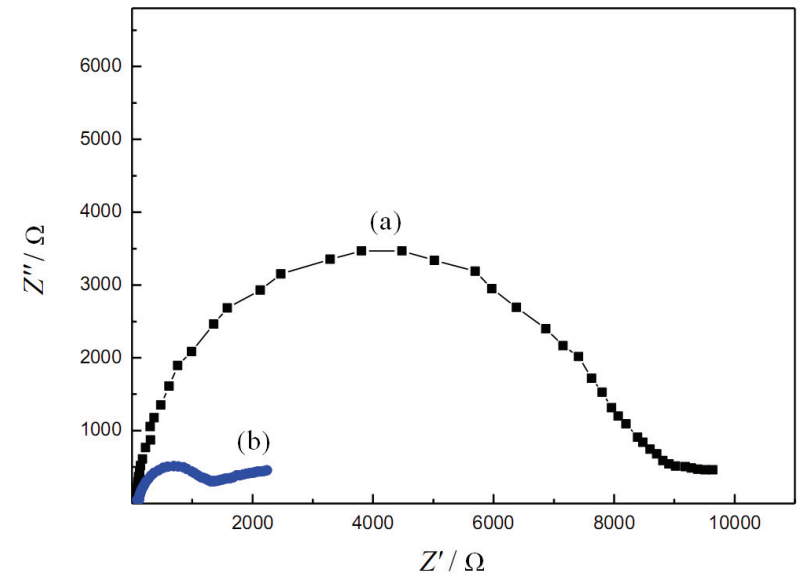

Figure 3. EIS for CPE (a) and $\mathrm{BPPF}_{6} / \mathrm{CPE}(\mathrm{b})$ in $1.0 \times 10^{-3}$ mol dm ${ }^{-3}\left[\mathrm{Fe}(\mathrm{CN})_{6}\right]^{3-14-}+0.10 \mathrm{~mol} \mathrm{dm}^{-3} \mathrm{KCl}$. The frequency range is $1-10^{5} \mathrm{~Hz}$.

In the meanwhile, the influence of the square root of scanning rate $\left(v^{1 / 2}\right)$ on the oxidation peak currents $\left(I_{\mathrm{p}}\right)$ of $3.0 \times 10^{-4} \mathrm{~mol} \mathrm{dm}^{-3} \mathrm{NAA}$ at both $\mathrm{CPE}$ and $\mathrm{BPPF}_{6} / \mathrm{CPE}$ were verified in the scanning rate range from 10 to $1000 \mathrm{mV} \mathrm{s}^{-1}$, respectively. The peak currents of NAA increase with the increasing of the square root of scanning rates on both electrodes, and there is a good linear relationship between $I_{\mathrm{p}}$ and $v^{1 / 2}$ respectively. The regression equations are $I_{\mathrm{p}} / \mu \mathrm{A}=-1.397+0.6682 v^{1 / 2} /$ $\mathrm{mV}^{1 / 2} \mathrm{~s}^{-1 / 2}(R=0.9987)$ and $I_{\mathrm{p}} / \mu \mathrm{A}=-3.383+1.675$ $v^{1 / 2} / \mathrm{mV}^{1 / 2} \mathrm{~s}^{-1 / 2}(R=0.9986)$, indicating that its electrochemical oxidation reaction at both $\mathrm{CPE}$ and $\mathrm{BPPF}_{6} / \mathrm{CPE}$ are a diffusion-controlled electrode reaction process.

\section{EIS Characterization}

Electrochemical impedance spectroscopy (EIS) is a useful tool for reflecting the interface properties of the modified electrodes ${ }^{17}$ by using $\left[\mathrm{Fe}(\mathrm{CN})_{6}\right]^{3-/ 4-}$ redox couples as an electrochemical probe (the experimental results were shown by curves in Figure 3). Generally, the diameter of the semicircle was usually equal to the electron transference resistance (Ret). ${ }^{18}$ Therefore, Ret can be used to describe the interface properties of the electrodes. From the curves in Figure 3 it showed that the impedance spectra in the form of Nyquist diagrams for both of the electrodes with the frequencies from $1 \mathrm{~Hz}$ to $100 \mathrm{kHz}$, and the whole profile for $\mathrm{BPPF}_{6} / \mathrm{CPE}$ exhibited a quasi-semicircle portion with much smaller diameters (curve b), which was attributed to the good ionic conductivity of ILs in the carbon paste and the least charge transfer resistance. ${ }^{11,12}$ For the CPE an obvious semicircle was observed (curve a), and the diameter of the semicircle (curve a) was much larger than that of $\mathrm{BPPF}_{6} / \mathrm{CPE}$. This might be due to the presence of nonconductive paraffin oil in the carbon paste,

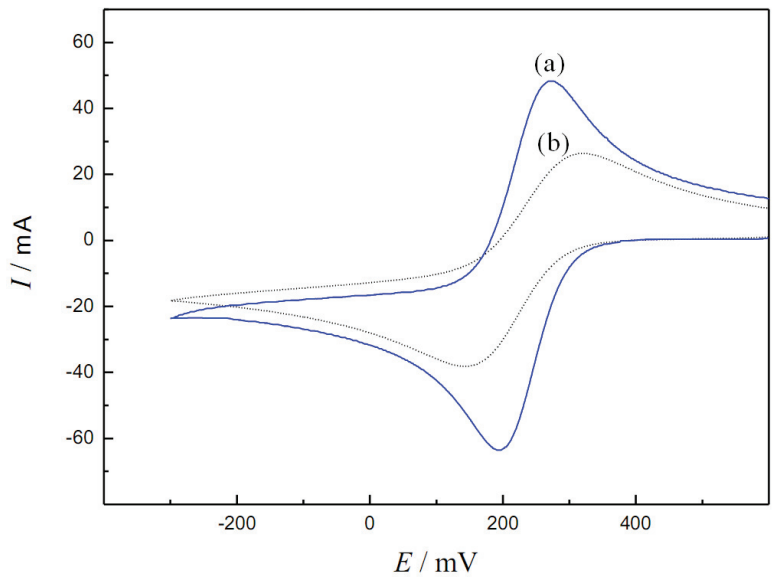

Figure 4. $\mathrm{CV}$ curves of $\mathrm{CPE}$ (a) and $\mathrm{BPPF}_{6} / \mathrm{CPE}$ (b) in $5.0 \times$ $10^{-3} \mathrm{~mol} \mathrm{dm}^{-3} \mathrm{~K}_{3}\left[\mathrm{Fe}(\mathrm{CN})_{6}\right]+1.0 \mathrm{~mol} \mathrm{dm}{ }^{-3} \mathrm{KCl}$. Scan rate: 50 $\mathrm{mV} \mathrm{s}^{-1}$.

which played an important role in hindering the electron transfer and made it more difficult for the electron transfer reaction to take place, thus increased the resistance to $\left[\mathrm{Fe}(\mathrm{CN})_{6}\right]^{3-/ 4-}$ redox couple. ${ }^{19}$ The results indicated that the $\mathrm{BPPF}_{6} / \mathrm{CPE}$ showed the properties much different from that of the CPE. This superiority of $\mathrm{BPPF}_{6} / \mathrm{CPE}$ can also be shown by cyclic voltammograms of $5.0 \times 10^{-3} \mathrm{~mol} \mathrm{dm}^{-3} \mathrm{~K}_{3}\left[\mathrm{Fe}(\mathrm{CN})_{6}\right]$ in $1.0 \mathrm{~mol} \mathrm{dm}^{-3} \mathrm{KCl}$ at both $\mathrm{CPE}$ and $\mathrm{BPPF}_{6} / \mathrm{CPE}$ in Figure 4. From the curves in Figure 4 it can be seen that the oxidation and reduction peak current at $\mathrm{BPPF}_{6} / \mathrm{CPE}$ were all increased about 2 times larger than that at CPE. The peak potential difference between oxidation peak potential and reduction peak potential at $\mathrm{BPPF}_{6} / \mathrm{CPE}$ was $77 \mathrm{mV}$. It was $100 \mathrm{mV}$ smaller than that at CPE.

\section{The Effect of Experimental Conditions on the Peak Currents and Potentials}

The types of the supporting electrolytes played an important role in the voltammetric responses of NAA. The current responses of $3.0 \times 10^{-4} \mathrm{~mol} \mathrm{dm}^{-3}$ NAA were evaluated in different supporting electrolytes such as aqueous $\mathrm{Na}_{2} \mathrm{HPO}_{4}-\mathrm{NaH}_{2} \mathrm{PO}_{4}, \quad \mathrm{NaAc}-\mathrm{HAc}, \mathrm{NaNO}_{3}$, $\mathrm{Na}_{2} \mathrm{SO}_{4}, \mathrm{NaCl}$ and $\mathrm{NaClO}_{4}$ solutions. The experimental results showed that a well-defined electrochemical behavior could be obtained in aqueous $\mathrm{NaClO}_{4}$ solution. And then, its voltammetric behaviors were compared in $0.010-0.50 \mathrm{~mol} \mathrm{dm}{ }^{-3}$ aqueous $\mathrm{NaClO}_{4}$ solutions. The experiment results showed that in $0.10 \mathrm{~mol} \mathrm{dm}^{-3}$ $\mathrm{NaClO}_{4}$ NAA has a well-defined electrochemical behavior. Therefore, $0.10 \mathrm{~mol} \mathrm{dm}{ }^{-3}$ aqueous $\mathrm{NaClO}_{4}$ solution was selected.

The effects of solution $\mathrm{pH}$ on NAA electrochemical responses were investigated in the range of $\mathrm{pH}=2.0$ to 10.0 (experimental results was shown curves in Figure 5). From curves in Figure 5, it can be seen that its 


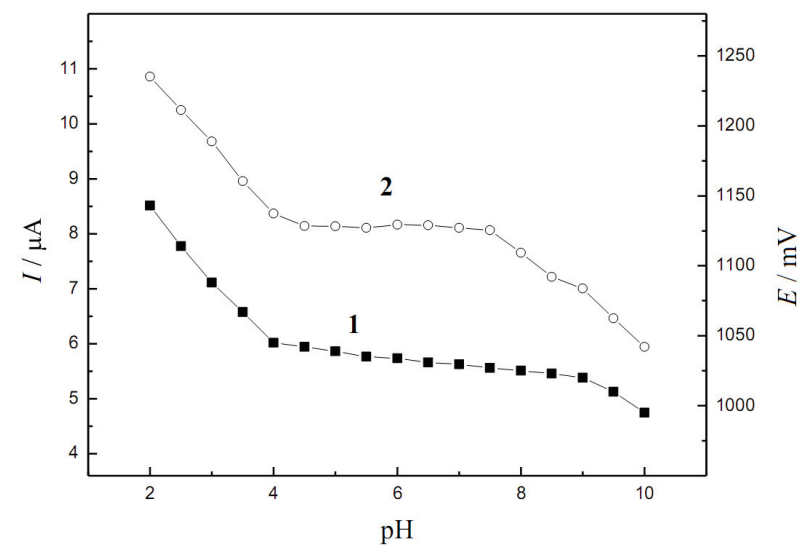

Figure 5. Effect of $\mathrm{pH}$ on $E_{\mathrm{p}}(1)$ and $I_{\mathrm{p}}(2)$ of NAA, $c(\mathrm{NAA})=$ $3.0 \times 10^{-4} \mathrm{~mol} \mathrm{dm}^{-3}$.

oxidation peak potentials decreased gradually with the increasing $\mathrm{pH}$ from 2.0 to 4.5 , and with a linear regression equation as $E_{\mathrm{p}}=-0.049 \mathrm{pH}+1.2372$ (shown curve 1 in Figure 5), and a correlation coefficient of 0.9976. This slope $(-0.049 \mathrm{~V} / \mathrm{pH})$ was very close to the theoretical slope value $(-0.059 \mathrm{~V} / \mathrm{pH})$ of the Nernst equation, which indicated that the electron-transfer number and the number of protons taking part in this electrode reaction process was the same. From our previous work ${ }^{15}$ we know $n=2$, thus $m=2$. This result indicated that there is a two-electron transfer accompanied with twoprotons participating in this electrode reaction process. But from $\mathrm{pH}=4.5$ to 9.0 , the oxidation peak potential was kept almost constant, and from $\mathrm{pH}=9.0$ to 10.0 , there was a little decrease. At the same time, the curve of peak current versus $\mathrm{pH}$ kept almost similar tendency with the curve of peak potential versus $\mathrm{pH}$. That is, peak currents decreased in $\mathrm{pH}=2.0$ to 4.5 and 7.5 to 10.0 , and there was nearly no significant variation from $\mathrm{pH}=$ 4.5 to 7.5. Therefore from our electrochemical experimental results it can be concluded that NAA electrochemical oxidation reaction is an irreversible and diffusion-controlled electrode reaction process with a loss of $2 \mathrm{e}^{-}$and $2 \mathrm{H}^{+}$.

\section{Electrochemical Kinetics}

\section{The Charge-transfer Coefficient $\alpha$}

The influence of the scanning rates $(v)$ on the oxidation peak potentials $\left(E_{\mathrm{p}}\right)$ of $3.0 \times 10^{-4} \mathrm{~mol} \mathrm{dm}^{-3} \mathrm{NAA}$ at CPE and $\mathrm{BPPF}_{6} / \mathrm{CPE}$ were also investigated in the range from 10 to $1000 \mathrm{mV} \mathrm{s}^{-1}$, respectively. From the experiment results, we can see that with the increasing of $v$ the oxidation peak potential was positively shifted. The Tafel slope $b$ was determined using the following equation valid for a totally irreversible diffusion-controlled process: ${ }^{20}$

$$
E_{\mathrm{p}}=(b \log v) / 2+\text { constant }
$$

On the basis of Equation (1), the slope of $E_{\mathrm{p}}$ versus $\log v$ plot is $b / 2$, where $b$ indicates the Tafel slope. The linear regression equation was got as $E_{\mathrm{p}} / \mathrm{mV}=$ $944.3+46.60 \log \left[v / \mathrm{mV} \mathrm{s}^{-1}\right](R=0.9988)$ on CPE and $E_{\mathrm{p}} / \mathrm{mV}=917.9+64.15 \log \left[v / \mathrm{mV} \mathrm{s}^{-1}\right](R=0.9989)$ on $\mathrm{BPPF}_{6} / \mathrm{CPE}$. The slope of $E_{\mathrm{p}}$ versus $\log v$ plot was found to be $46.60 \mathrm{mV}$ and $64.15 \mathrm{mV}$, respectively, so, $b=2 \times$ $46.60 \mathrm{mV}$ and $b=2 \times 64.15 \mathrm{mV}$. While $b=2.303 R T /$ $[n(1-\alpha) F]$, the number of electrons involved in the electrochemical oxidation reaction of NAA is $2,{ }^{15} F$ is the Faraday's constant $\left(\mathrm{C} \mathrm{mol}^{-1}\right)$, and the other symbols have their usual meanings, so it gives the chargetransfer coefficient $\alpha$ as 0.68 at $\mathrm{CPE}$ and 0.77 at $\mathrm{BPPF}_{6} / \mathrm{CPE}$, respectively.

\section{Diffusion Coefficient D}

The electrode surface area for the bare CPE and $\mathrm{BPPF}_{6} / \mathrm{CPE}$ can be determined by the slope of the curves of $Q$ versus $t^{1 / 2}$ by chronocoulometry $(\mathrm{CC})^{21}$ using $5.0 \times 10^{-3} \mathrm{~mol} \mathrm{dm}^{-3}\left[\mathrm{Fe}(\mathrm{CN})_{6}\right]^{3-}$ as model compound (the diffusion coefficient $D$ of $\left[\mathrm{Fe}(\mathrm{CN})_{6}\right]^{3-}$ is $7.6 \times 10^{-6} \mathrm{~cm}^{2} \mathrm{~s}^{-1}$ ) based on Equation (2)

$$
Q=2 n F A C \sqrt{D t / \pi}+Q_{\mathrm{dl}}+Q_{\mathrm{ads}}
$$

From those, the surface area $(A)$ of the CPE and $\mathrm{BPPF}_{6} / \mathrm{CPE}$ can be calculated, and then, according to the slope of the linear relationship between $Q$ and $t^{1 / 2}, D$ can be determined also. From the slop as $1.042 \times 10^{-5}$, it can gives a diffusion coefficient $D$ as $6.6 \times 10^{-6} \mathrm{~cm}^{2} \mathrm{~s}^{-1}$.

\section{The Electrode Reaction Rate Constant $\mathrm{k}_{f}$}

The electrode reaction rate constant $\left(k_{\mathrm{f}}\right)$ can be determined by Chronoampertry (CA) using the following equation $^{21}$

$$
I(t)=n F A k_{\mathrm{f}} C[1-2 H \sqrt{t / \pi}]
$$

In which, $H=k_{\mathrm{f}} / D_{\mathrm{Ox}}^{1 / 2}+k_{\mathrm{b}} / D_{\mathrm{Rd}}^{1 / 2}$. For the totally irreversible electrochemical reaction, the value of $k_{\mathrm{b}}$ is 0 , therefore, when $t$ approaches to 0 , the plot of $I(t)$ versus $t^{1 / 2}$ gives a good straight line. So, $k_{\mathrm{f}}$ can be calculated from the intercept of CA curve, the resulting values of $k_{\mathrm{f}}$ are $0.82 \times 10^{-3} \mathrm{~cm} \mathrm{~s}^{-1}(\mathrm{CPE})$ and $0.96 \times 10^{-3}$ $\mathrm{cm} \mathrm{s}^{-1}\left(\mathrm{BPPF}_{6} / \mathrm{CPE}\right)$.

\section{Order Number of Electrochemical Reaction}

Based on electrochemical kinetics, ${ }^{22}$ the logarithm of the oxidation peak currents with logarithm of NAA concentrations was plotted with the regression equation as $\log \left[I_{\mathrm{p}} / \mu \mathrm{A}\right]=-0.9362+1.057 \log \left[c_{\mathrm{NAA}} /\left(10^{-3} \mathrm{~mol}\right.\right.$ $\left.\left.\mathrm{dm}^{-3}\right)\right]$ in the NAA concentration range of $2.0 \times 10^{-5}$ to $4.0 \times 10^{-4} \mathrm{~mol} \mathrm{dm}{ }^{-3}, R=0.9976$. The straight line with the slope of 1.057 , closing near to1, was obtained, which implies that the electrochemical oxidation reaction of $\mathrm{NAA}$ on $\mathrm{BPPF}_{6} / \mathrm{CPE}$ is a quasi-first order reaction. 


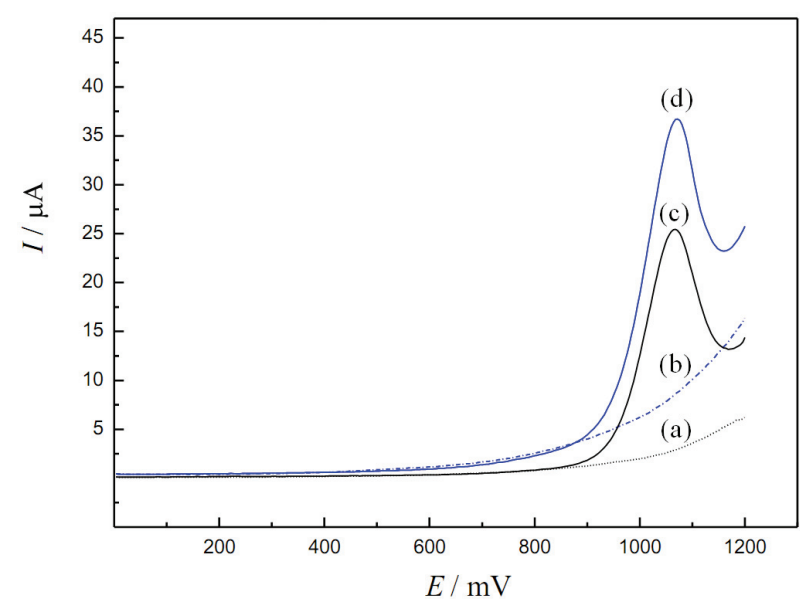

Figure 6. $\mathrm{SWV}$ curves of $\mathrm{CPE}(\mathrm{a})$ and $\mathrm{BPPF}_{6} / \mathrm{CPE}(\mathrm{b})$ in 0.10 mol $\mathrm{dm}^{-3} \mathrm{NaClO}_{4}$ in the absence and the presence of $3.0 \times 10^{-4} \mathrm{~mol} \mathrm{dm}^{-3} \mathrm{NAA}$ at $\mathrm{CPE}(\mathrm{c})$ and $\mathrm{BPPF}_{6} / \mathrm{CPE}(\mathrm{d})$.

\section{Electrochemical Determination Application}

Square-wave Voltammetric (SWV) Behaviors The SWV behaviors of NAA at $\mathrm{BPPF}_{6} / \mathrm{CPE}$ under the optimized experimental conditions (the pulse amplitude $45 \mathrm{mV}$, frequency $55 \mathrm{~Hz}$, and the scanning potential increment $4 \mathrm{mV}$ ) showed that its oxidation potential at $\mathrm{BPPF}_{6} / \mathrm{CPE}$ (curve $\mathrm{d}$ in Figure 6) almost keep constant as that at CPE (curve $\mathrm{c}$ in Figure 6), but its oxidation currents are enhanced greatly, about $50 \%$ increased. This is in a quite good agreement with that of CV. The relationship between oxidation peak currents for NAA and its concentration were investigated at BPPF6/CPE. The linear calibration curves are obtained over the concentration range of $2.0 \times 10^{-5}$ to $4.0 \times 10^{-4} \mathrm{~mol} \mathrm{dm}^{-3}$ with a linear regression equation of $I_{\mathrm{p}} / \mu \mathrm{A}=-1.760+$ $71.00 \mathrm{c} /\left(10^{-3} \mathrm{~mol} \mathrm{dm}^{-3}\right)$, and a correlation coefficient of 0.9989 , and the detection limit $(\mathrm{S} / \mathrm{N}=3)$ as $1.690 \times 10^{-7}$ $\mathrm{mol} \mathrm{dm}{ }^{-3}$.

\section{Interferences}

The influences of various possible interferents were also examined by analyzing a standard solution of NAA. A number of foreign inorganic compounds existing in commercial formulations such as 1000 times of $\mathrm{K}^{+}, \mathrm{Na}^{+}, \mathrm{Zn}^{2+}, \mathrm{NO}_{3}^{-}, \mathrm{SO}_{4}^{2-}, \mathrm{NH}_{4}^{+}$, and $\mathrm{Cl}^{-}$were studied, within the tolerance limit of $\pm 5.0 \%$, all of these foreign compounds didn't affect NAA currents response.

\section{The Stability and the Reproducibility}

After the modified electrode was stored in the room temperature for ten days, the current response obtained was within less than $\pm 5 \%$, which can be attributed to the excellent stability of the modified electrode. Ten times of the parallel determinations were made in order to inspect the reproducibility of the electrode. The experimental results shown that the determinations results nearly keep constant and the relative standard deviation (RSD) as $2.8 \%$ which showed a good reproducibility for the modified electrode.

\section{Electrochemical Determination}

In order to confirm the application of the proposed method, the NAA content determinations in both commercial formulations and some soil samples were performed, respectively. The commercial formulations of 1-naphthaleneacetic acid and the soil samples (the soil samples were collected from different farmlands and treated as previously reported $)^{9}$ were determined by SWV. Determinations of NAA content in the two kinds of the samples were carried out by standard addition, the experimental result was shown in Table 1. An acceptable reproducibility with a relative standard deviation of $1.2-1.8 \%$ of commercial formulations was obtained for six parallel measurements, the relative standard deviation of $1.6-2.7 \%$ for soil samples. In addition, the recoverage on the basis of the two method were in the range of $97.43-104.7 \%$ (commercial formulation) and $100.3-102.6 \%$ (soil samples). The result indicated that the proposed one can be used as an effective electrochemical quantitative determination of NAA in both commercial formulations and the soil samples.

\section{CONCLUSIONS}

An IL/CPE, using $N$-butylpyridinium hexafluorophosphate as modifier, had been fabricated and charac-

Table 1. Determination results of the samples $(n=6)$

\begin{tabular}{|c|c|c|c|c|c|c|}
\hline Samples & Labeled & Determined / g & RSD / \% & Added / g & Determined / g & Recoverage / \% \\
\hline \multirow{3}{*}{$\begin{array}{l}\text { Commercial } \\
\text { sample }\end{array}$} & \multirow{3}{*}{$0.5000 \mathrm{~g} / \mathrm{pack}$} & 0.4966 & 1.8 & 0.3571 & 0.3740 & 104.7 \\
\hline & & 0.4915 & 1.2 & 0.5000 & 0.4872 & 97.43 \\
\hline & & 0.5088 & 1.5 & 0.6429 & 0.6309 & 98.13 \\
\hline \multirow{4}{*}{ Soil sample } & & Determined $/ \mu \mathrm{g} \mathrm{g}^{-1}$ & $\mathrm{RSD} / \%$ & Added / $\mu \mathrm{g} \mathrm{g}^{-1}$ & Determined $/ \mu \mathrm{g} \mathrm{g}^{-1}$ & Recoverage / \% \\
\hline & & 13.57 & 1.7 & 0.9311 & 0.9359 & 100.5 \\
\hline & & 13.02 & 1.6 & 1.3035 & 1.3376 & 102.6 \\
\hline & & 13.31 & 2.7 & 1.6756 & 1.6811 & 100.3 \\
\hline
\end{tabular}


terized by EIS and voltammetry. The electrochemical behaviors of NAA at two electrodes were investigated. Compared with its currents response at $\mathrm{CPE}$, the electrochemical sensitivity of NAA at the modified electrode was improved remarkably. At the same time, the electrochemical kinetics parameters were determined at both electrodes. The proposed method was further used for the determination of NAA content with the satisfactory results. The oxidation peak currents increased linearly with the concentration of NAA in the range from $2.0 \times 10^{-5}$ to $4.0 \times 10^{-4} \mathrm{~mol} \mathrm{dm}^{-3}$ with the detection limit $(\mathrm{S} / \mathrm{N}=3)$ as $1.690 \times 10^{-7} \mathrm{~mol} \mathrm{dm}^{-3}$ under the optimized experiment conditions. The method was successfully applied in the determination of NAA content in both commercial formulations and the soil samples.

\section{REFERENCES}

1. G. Zweig, D. L. Gutnick, R. Gulli, T. E. Archer, and H. T. Hartmann, J. Agric. Food. Chem. 12 (1964) 59-61.

2. B. Maiti, S. R. Desai, and T. S. Krishnamoorthy, Analyst 113 (1988) 667-668.

3. J. L. Vilchez, R. Blanc, and A. Navalón, Talanta 45 (1997) 105-111

4. A. Navalón, R. Blanc, and J. L. Vilchez, Mikrochim. Acta 126 (1997) 33-38.

5. J. A. M. Pulgarín, P. F. López, L. F. G. Bermejo, and F. M. Alfonso, J. Agric. Food. Chem. 51 (2003) 6380-6385.
6. M. T. Fernández-Argüelles, B. Cañabate, A. Segura, J. M. Costa, R. Pereiro, A. Sanz-Medel, and A. Fernández, Talanta 66 (2005) 696-702.

7. B. F. Liu, X. H. Zhong, and Y. T. Lu, J. Chromatogr. A 945 (2002) 257-265.

8. M. Yuan, M. Zhang, and W. Kangjing, Chin. J. Chromatogr 15 (1997) 482-485.

9. S. F. Lü, Anal. Lett. 36 (2003) 1523-1534.

10. W. S. Huang, W.Y.Qu, and D. Z. Zhu, Bull. Korean Chem. Soc. 29 (2008) 1323-1326.

11. P. Wasserscheid and T. Welton (Eds.), Ionic Liquids in Synthesis, Wiley/VCH Verlag, Weinheim, 2003.

12. J. Dupont, R. F. de Souza, and P. A. .Z. Suarez, Chem. Rev. 102 (2002) 3667-3692.

13. L. H. Liu, C. Q. Duan, and Z. N. Gao, Croat. Chem. Acta 83 (2010) 409-414.

14. L. H. Liu and Z. N. Gao, Chin, J. Pharm. Anal 30 (2010) 438-442.

15. J. W. Chen, C. Q. Duan, and Z. N. Gao, Chem. Res. Appl. 23 (2011) 431-436.

16. N. Maleki, A. Safavi, and F. Tajabadi, Electroanal. 19 (2007) 2247-2250.

17. J. J. Feng, G. Zhao, J. J. Xu, and H. Y. Chen, Anal. Biochem. 342 (2005) 280-286.

18. E. Katz and I. Willner, Electroanal. 15 (2003) 913-947.

19. W. Sun, M. X. Yang, and K. Jiao, Anal. Bioanal. Chem. 389 (2007) 1283-1291.

20. S. M. Golabi and H. R. Zara, Electroanal. 11 (1999) 1293-1300.

21. R. N. Adams, Electrochemistry at Solid Electrodes, Marcel Dekker, New York, 1969, p. 220.

22. H. Q. Wu and Y. F. Li, Electrochemical kinetics, China Higher Education Press, Beijing, China, 1998, p.95-99. 